\title{
A Fungal Dirigent Protein Controls the Stereoselectivity of Multicopper Oxidase-Catalyzed Phenol Coupling in Viriditoxin Biosynthesis
}

\author{
Jinyu Hu, ${ }^{\dagger}$ Hang Li, ${ }^{\dagger}$ Yit-Heng Chooi ${ }^{\dagger}, *$ \\ †School of Molecular Sciences, University of Western Australia, Perth, Western Australia 6009, Australia.
}

\section{Supporting Information Placeholder}

\begin{abstract}
Paecilomyces variotii produces the antibacterial and cytotoxic $(M)$-viriditoxin (1) together with a trace amount of its atropisomer $(P)$-viriditoxin $\mathbf{1}^{\prime}$. Elucidation of the biosynthesis by heterologous pathway reconstruction in Aspergillus nidulans identified the multicopper oxidase (MCO) VdtB responsible for the regioselective 6,6'-coupling of semiviriditoxin (10), which yielded $\mathbf{1}$ and $\mathbf{1}^{\prime}$ at a ratio of $1: 2$. We further uncovered that VdtD, an $\alpha / \beta$ hydrolase-like protein lacking the catalytic serine, directs the axial chirality of the products. Using recombinant VdtB and VdtD as cell-free extracts from $A$. nidulans, we demonstrated that VdtD acts like a dirigent protein to control the stereoselectivity of the coupling catalyzed by VdtB to yield $\mathbf{1}$ and $\mathbf{1}^{\prime}$ at a ratio of 20:1. Furthermore, we uncovered a unique Baeyer-Villiger monooxygenase (BVMO) VdtE that could transform the alkyl methylketone side chain to methyl ester against the migratory aptitude.
\end{abstract}

Axially chiral biaryl natural products pose unique challenges for total synthesis due to the presence of both regiochemistry and stereochemistry at the biaryl axis. ${ }^{1}$ In nature, biaryl compounds are generated via intermolecular oxidative phenol coupling. For fungi, phenol coupling has been shown to be catalyzed by $\mathrm{P} 450$ monooxygenases or laccases/multicopper oxidases (MCOs). ${ }^{2-7}$ Although P450s have been shown to catalyze regio- and stereoselective phenol coupling, such as in biosynthesis of kotanin (Figure 1), 6 MCOs are not known to be able to control the axial chirality of biaryl products. ${ }^{2,} 7$ For example, an MCO with regioselective 8, 8' ortho-ortho coupling activity was identified from Talaromyces pinophilus in the biosynthesis of dinapinone A (Figure 1), albeit it is not stereoselective. ${ }^{2}$ In higher plants, the regio- and stereoselective coupling by MCOs are controlled by non-catalytic auxiliary proteins known as dirigent proteins, ${ }^{8-10}$ but no such protein had been uncovered in fungi to date. Recently, our group showed that the regio- and stereoselective double coupling of naphthols to form the two $\mathrm{C}$-C bridges in fungal perylenequinones (e.g. elsinochrome A in Figure 1) involved the close cooperation of an MCO and a berberine bridge enzyme-like oxidase. ${ }^{11}$ However, it was difficult to attribute the stereoselectivity to one or both enzymes due to the double coupling reactions could not be separated and the naphthol substrate is unstable.

Here, we focus on the investigation of the biosynthesis of a bisnaphthopyrone, viriditoxin (1), with a single C-C bridge between the two monomers. Compound $\mathbf{1}$ was first isolated from Aspergillus viridinutans, ${ }^{12}$ in which the structure was revised to 6,6'-bisnaphtho- $\alpha$ - pyrone with an $R$-configuration at the $6,6^{\prime}$ axis, which gives an $(M)$ helical conformation. ${ }^{13}$ Another fungus Paecilomyces variotii has been reported to produce $\mathbf{1}$ and its derivatives. ${ }^{14-16}$ Compound $\mathbf{1}$ possesses wide spectrum antibacterial activity and cytotoxicity against different cancer cell lines. ${ }^{17-19}$ It had been shown to inhibit the bacterial cell division protein FtsZ under certain conditions. 17, ${ }^{20}$ Despite the broad interest in $\mathbf{1}$ and established total synthesis routes, 21,22 little was known about the molecular basis of its biosynthesis.
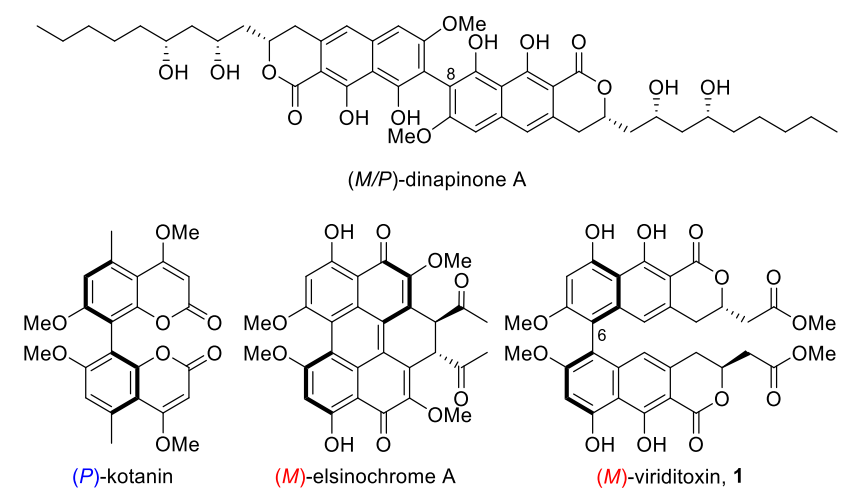

Figure 1. Examples of axially chiral biaryl natural products from fungi.

The genome of $P$. variotii strain CBS101075 (Figure 1) was recently sequenced, ${ }^{23}$ hence it was chosen for studying the biosynthesis of $\mathbf{1}$. The biosynthesis of $\mathbf{1}$ is hypothesized to start from a naphtho- $\alpha$-pyrone synthesized by non-reducing polyketide synthase (NR-PKS). BLASTp query using the NRPKS ElcA ${ }^{24}$ from the elsinochrome pathway, known to produce a similar naphthopyrone nor-toralactone, identified an NRPKS protein (ID 480069) with high similarity to ElcA in the $P$. variotii CBS101075 genome. Further analysis of its flanking region revealed a putative biosynthetic gene cluster of 9 genes (Figure 2), which encode all tailoring enzymes predicted to be required in the biosynthetic pathway of $\mathbf{1}$ (renamed as $v d t$ cluster). Analysis of the metabolite profile of $P$. variotii CBS101075 showed that it produces $(M)$-viriditoxin 1 and traces of the atropisomer $(P)$-viriditoxin $\mathbf{1}^{\prime}$ in approximately 20:1 ratio (Figure S1) suggesting the existence of regio- and stereo-selective coupling enzyme in viriditoxin biosynthesis. However, in the $v d t$ cluster, the only candidate gene encoding 
phenol coupling enzyme is $v d t B$ encoding an MCO, which have never been reported to be stereoselective. Therefore, the basis for the stereoselective para-para coupling in biosynthesis of 1 was enigmatic. Furthermore, the presence of $v d t E$ encoding a Baeyer-Villiger monooxygenase (BVMO) suggests the methyl ester in $\mathbf{1}$ could be formed via a Baeyer-Villiger oxidation. Such naturally occurring methyl ester-forming BVMOs are rare as methyl esters often exist as a minor co-product to the other more preferred regioisomer.25, 26 Thus, we set out to investigate the biosynthetic pathway by heterologous reconstruction in $A$. nidulans accompanied by in vitro characterization of selected enzymes either as purified recombinant proteins or cell-free lysate from A. nidulans expressing the corresponding proteins. Compounds obtained from the strains constructed were isolated and characterized by MS and NMR analyses (Table S4-S18). We employed the episomal yeastfungal artificial chromosome (YFAC) expression system we established previously ${ }^{11}$ and the A. nidulans strain L07890.27 Firstly, expression of $v d t A$ in $A$. nidulans resulted in the accumulation of $\mathbf{4}$ as expected. Following our hypothesized pathway, we then added $v d t C$ encoding $O$-methyltransferase with $v d t A$ in $A$. nidulans and the strain produces the methylated 5 (Figure $3 \mathrm{~A}$ and 4 ). Next, we co-expressed $v d t F$ encoding a short-chain dehydrogenase/reductase (SDR) with $v d t A / C$ in $A$. nidulans and resulted in the production of $\mathbf{6}$, suggesting that $\mathrm{VdtF}$ acts as a stereo-specific reductase converting the pyrone 5 to dihydropyrone 6 (Figure 3A and 4).

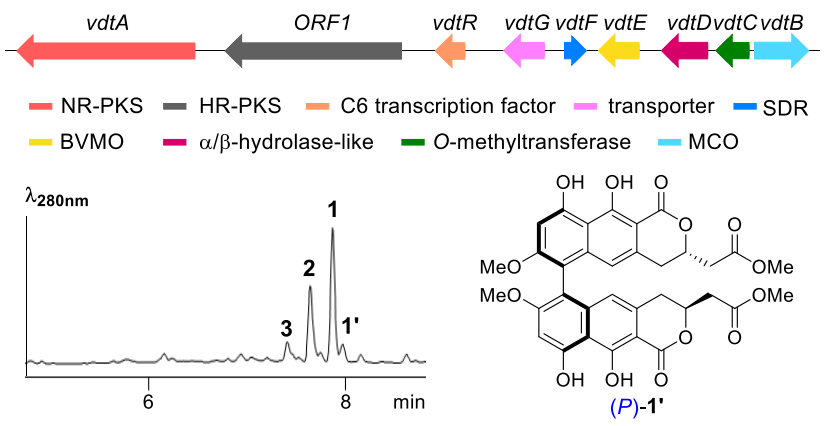

Figure 2. The $v d t$ cluster (see Table S3) and the metabolite profile of Paecilomyces variotii showing the production of $\mathbf{1}$ and atropisomer $\mathbf{1}^{\prime}$.

We next co-expressed $v d t E$ encoding the BVMO with $v d t A / C$ and $v d t A / C / F$ in $A$. nidulans. A. nidulans $v d t A / C / E$ produced the methyl ester derivative $\mathbf{8}$ and unexpectedly, together with a hydrolyzed carboxyl derivative $\mathbf{7}$. With $v d t F$, the $\mathbf{7 / 8}$ pair were converted to $\mathbf{9 / 1 0}$ in $A$. nidulans $v d t A / C / E / F$ (Figure $3 \mathrm{~A})$. The results confirmed the role of VdtE as BVMO, but confusion remained as BVMOs have never been reported to possess hydrolyzing activity. ${ }^{28,29}$ To further characterize the function of VdtE, we purified VdtE as maltose binding protein (MBP)-tagged recombinant protein from E. coli BL21(DE3) for in vitro enzymatic assay using $\mathbf{5}$ as substrate. NADH and NADPH were both tested as reducing cofactors. In the presence of FAD, VdtE was able to utilize NADPH to fully convert 5 to $\mathbf{8}$ and $\mathbf{6}$ to $\mathbf{1 0}$ (Figure $3 \mathrm{~B}$ and S4C). Thus, it is conclusive that VdtE is a methyl ester-forming BVMO and the formation of the carboxyl derivatives $\mathbf{7}$ and $\mathbf{9}$ and in A. nidulans is likely due to endogenous hydrolase activity.

We initially proposed that the hydrolase VdtD could hydrolyze the methyl ester and hence its co-expression would lead to an increase in the carboxyl acid derivatives. Interestingly, co-expression of $v d t D$ with $v d t A / C / E$ resulted in in- crease in the methyl ester 8 instead compared to without $v d t D$, while co-expression of $v d t D$ along with $v d t A / C / E / F$ resulted in loss of $\mathbf{9}$ and exclusive accumulation of the methyl ester $\mathbf{1 0}$ (Figure 3A). This observation of the shifts toward the methyl ester derivatives in the presence of VdtD (Figure $3 \mathrm{~A}$ ) raised the question about the function of the putative hydrolase VdtD in viriditoxin biosynthesis. The results suggest that instead of functioning as a hydrolase, VdtD possesses an opposite role it protects the methyl ester from being hydrolyzed by the endogenous hydrolases, perhaps via binding to the compounds. Analysis of VdtD protein sequence identified a mutation in the catalytic triad Ser-(Glu/Asp)-His, ${ }^{30}$ in which the important serine residue was mutated to aspartate (Figure S5).
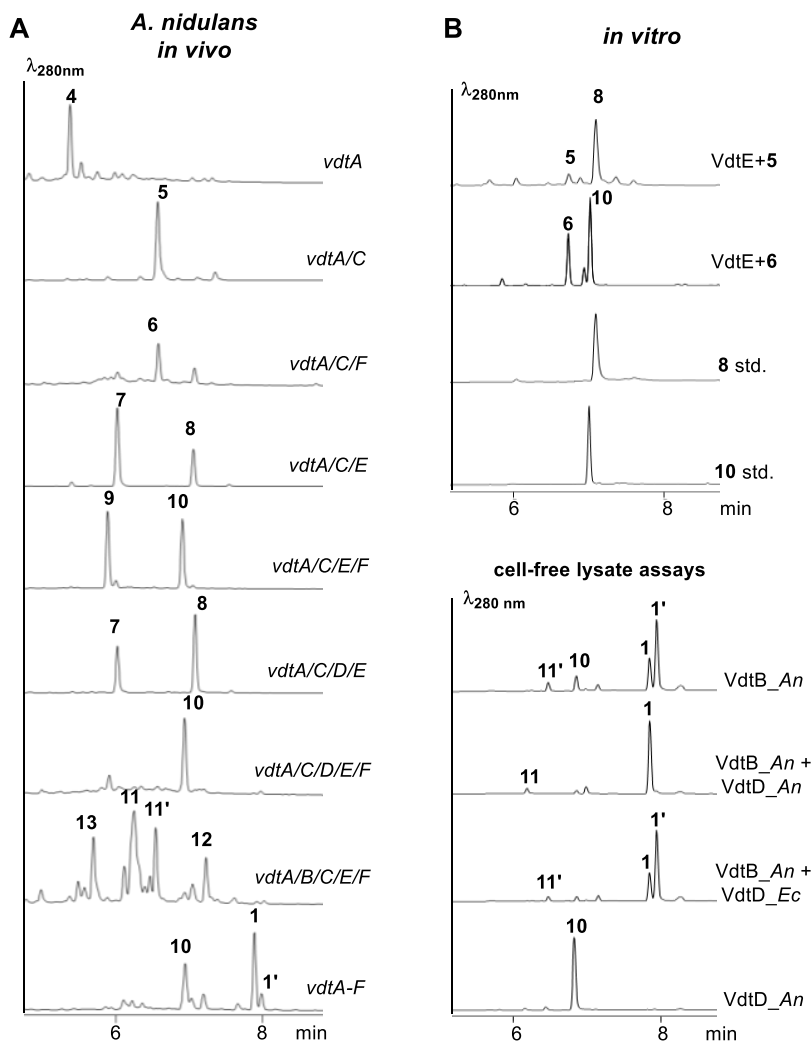

Figure 3. LC-DAD-MS traces for (A) heterologous reconstruction of $v d t$ cluster and (B) in vitro reactions of $\mathrm{VdtE}$ with $\mathbf{5}$ and $\mathbf{6}$ (top) and VdtB/VdtD cell-free lysates with $\mathbf{1 0}$ (bottom). An, $A$. nidulans cell-free lysates; $E c$, recombinant VdtD purified from E. coli.

Next, $v d t B$ encoding the MCO was co-expressed with other $v d t$ genes. Co-expression of $v d t A-F$ in $A$. nidulans reconstituted the production of $\mathbf{1 / \mathbf { 1 } ^ { \prime }}$ at a 20:1 ratio similar to P. variotii. However, in the absence of $v d t D$ encoding the non-catalytic hydrolase-like protein, $A$. nidulans $v d t A / B / C / E / F$ produced multiple new peaks with UV-vis spectra similar to bisnathopyrones along with traces of $\mathbf{1}$ and $\mathbf{1}^{\prime}$ (Figure $3 \mathrm{~A}$ and S2). Among them, the major products that we were able to isolate are a pair of atropisomeric carboxyl bisnaphthopyrone deriva tives $\mathbf{1 1}$ and 11' and two heterodimers 12 and 13 in $(P)$ conformation (Figure 5). It appears that in the absence of VdtD, VdtB lost the stereoselectivity and to a certain extent, the substrate fidelity as well. The presence of $\mathbf{1 3}$ also suggests that the methylation by VdtC could occur after VdtE and VdtF-catalyzed reactions. The carboxylic acids could be explained by the endogenous 


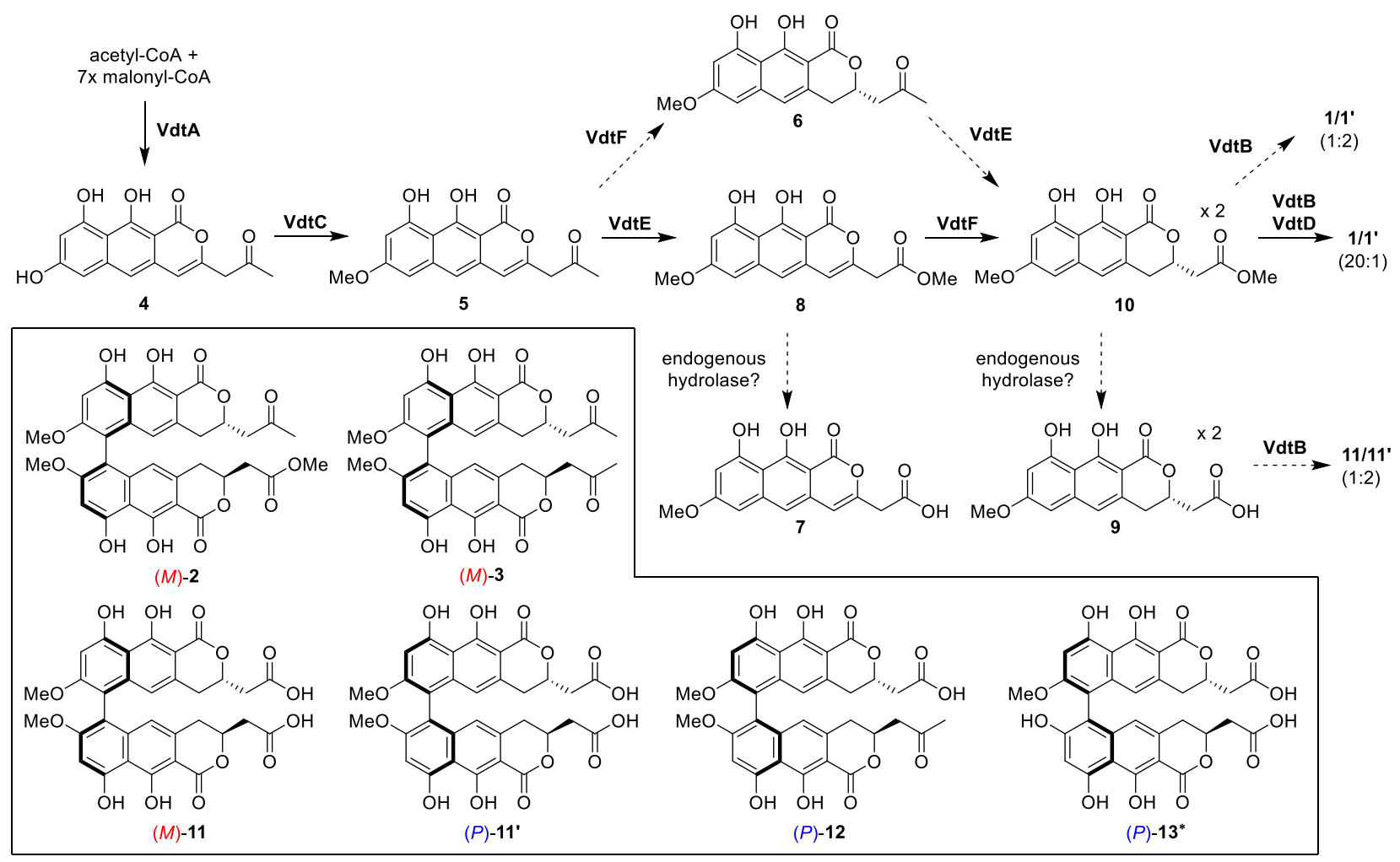

Figure 4. Elucidated biosynthetic pathway and structures of bisnaphthopyrone compounds isolated (box). * indicates proposed structure for 13.

hydrolase activity in the A. nidulans host and the absence of VdtD, which has been implicated above to have protective effects on the methyl esters against hydrolysis. Lastly, we showed that addition of ORF1 encoding a highly-reducing (HR-PKS) resulted in no changes in the metabolic profile (Figure $\mathrm{S1B}$ ), indicating that it is not involved in the biosynthesis of 1.

To corroborate the function of VdtB and VdtD, we attempted to characterize the proteins in vitro. Our attempts to express the proteins in E. coli was unsuccessful for VdtB. Given the success in the recent study using cell-free extract to reconstitute the activity of a fungal $M C O,{ }^{2}$ we took a similar approach. Cell-free extracts containing VdtB and VdtD were prepared separately from $A$. nidulans expressing the corresponding gene. Recombinant VdtD from $E$. coli was also included in the assays. The napthopyrone methyl ester $\mathbf{1 0}$ was used as the substrate. As shown in Figure 3B, VdtB alone slightly favored the production of $\mathbf{1}^{\prime}$ over $\mathbf{1}$ at a ratio of 2:1. When 10 was assayed together with VdtB and VdtD cell lysates, 1 was exclusively produced with a trace amount of the corresponding $(M)$ carboxyl derivative 11. Likewise, when the carboxylic acid monomer 9 was tested, VdtD altered the stereoselectivity of the oxidative coupling to favor the $(M) \mathbf{1 1}$ over $(P)$ 11', but less drastically (Figure S4B). The MBPtagged VdtD from $E$. coli had no influence on the stereoselectivity of the VdtB-catalyzed coupling, suggesting either that the recombinant protein was incorrectly folded or post-translational modification is required for its activity. The in vitro data clearly demonstrated the role of VdtB as a $6,6^{\prime}$ regioselective coupling $\mathrm{MCO}$ and that VdtD controls the stereoselectivity in the formation of $(M)-\mathbf{1}$.

In summary, we have uncovered a unique methyl esterforming BVMO and a novel non-catalytic auxiliary protein that controls the stereoselectivity of MCO-catalyzed phenol cou- pling. The exclusive methyl ester formation catalyzed by VdtE is intriguing, as the formation of methyl ester required the migration of the less nucleophilic carbon during the Criegee rearrangement, against the migratory aptitude (Figure S6A). ${ }^{25}$, 26,28 This is the first report of a naturally-occurring methyl ester-forming BVMO. Recently, directed evolution has been used to alter the regioselectivity of a BVMO TmCHMO to generate methyl ester, ${ }^{31}$ in which the reversal in regioselectivity was attributed to the change in conformation of the Criegee intermediate in the binding pocket. A unique fungal BVMO capable of inserting oxygen on both sides of a carbonyl to form a carbonate has also been discovered. ${ }^{32}$ Together, they highlight the catalytic prowess of fungal BVMOs.

More significantly, we uncovered that VdtD exerts control over the stereoselectivity of the $6,6^{\prime}$ regioselective coupling catalyzed by the MCO VdtB. While preparing this manuscript for submission, another group has independently demonstrated the regioselective coupling activity of the VdtB homo$\log (\mathrm{Av}-\mathrm{VirL})$ from another producer of $\mathbf{1}$ A. virinidutans. ${ }^{33}$ Using cell-free extract and a non-native substrate semivioxanthin, the corresponding dimer was produced but with a higher ratio of the incorrect $(P)$ atropisomer. This corresponds to our study that VdtD (or its homolog Av-VirE) is required for controlling the stereoselectivity of the MCO. Indeed, both VdtD and Av-VirE lack the conserved catalytic serine found in $\alpha / \beta$ hydrolases, albeit we have found other close homologs with the catalytic triad remains intact (Figure S5), suggesting that the mutation was acquired relatively recently. Therefore, functionally, VdtD/Av-VirE is analogous to the dirigent protein found in plants, which are non-catalytic proteins that could orient phenoxy radicals to direct stereospecific formation of biaryl products. ${ }^{8-10}$ The structure of the plant dirigent proteins have been solved and was shown to adopt an eight-stranded $\beta$-barrel with a large hydrophobic cavity.34,35 
Meanwhile, fungal lipocalin-like proteins predicted to have $\beta$ barrel fold have been shown to direct the stereochemistry of Diels-Alder reactions, ${ }^{36-38}$ some of which are considered as Diels-Alderases. ${ }^{39}, 40$ In contrast, VdtD belongs to the $\alpha / \beta$ hydrolase family and is related to fungal carboxyesterases/lipases (Figure S5). Structural and protein interaction studies will be required to understand the mechanism of the stereocontrol. To our knowledge, VdtD is the first dirigent protein reported in fungi that directs the stereoselectivity of an MCO. As gene encoding such $\alpha / \beta$ hydrolase-like dirigent protein is not co-located with MCO gene in some of the fungal biosynthetic gene clusters encoding biaryl compounds, 33 it is possible that there are other mechanisms for controlling the stereoselectivity of MCOs in fungi. This study expands the enzymatic tools for phenol oxidative coupling and BaeyerVilliger oxidation.

\section{ASSOCIATED CONTENT}

\section{Supporting Information}

Experimental details, supplementary figures and spectroscopic data.

The Supporting Information is available free of charge on the ACS Publications website.

\section{AUTHOR INFORMATION}

\section{Corresponding Author:}

*yitheng.chooi@uwa.edu.au

\section{Notes}

The authors declare no competing financial interests.

\section{ACKNOWLEDGMENT}

Y-HC is an Australian Research Council (ARC) Future Fellow. $\mathrm{JH}$ and HL are supported by Australian International Postgraduate Research Scholarships. NMR and HR-MS were performed at the UWA Centre for Microscopy, Characterization and Analysis (CMCA). Special thanks to A/Prof. Alexander Idnurm for providing the P. variotii CBS101075 and to Dr Andrew Piggott for helpful discussion.

\section{REFERENCES}

(1). Bringmann, G.; Gulder, T.; Gulder, T. A.; Breuning, M., Atroposelective total synthesis of axially chiral biaryl natural products. Chem. Rev. 2011, 111 (2), 563-639.

(2). Kawaguchi, M.; Ohshiro, T.; Toyoda, M.; Ohte, S.; Inokoshi, J.; Fujii, I.; Tomoda, H., Discovery of a Fungal Multicopper Oxidase That Catalyzes the Regioselective Coupling of a Tricyclic Naphthopyranone To Produce Atropisomers. Angew. Chem., Int. Ed. 2018, 57 (18), 51155119.

(3). Griffiths, S.; Mesarich, C. H.; Saccomanno, B.; Vaisberg, A.; De Wit, P. J.; Cox, R.; Collemare, J., Elucidation of cladofulvin biosynthesis reveals a cytochrome P450 monooxygenase required for anthraquinone dimerization. Proc. Natl. Acad. Sci. U. S. A. 2016, 113 (25), 6851-6856.

(4). Frandsen, R. J.; Schutt, C.; Lund, B. W.; Staerk, D.; Nielsen, J.; Olsson, S.; Giese, H., Two novel classes of enzymes are required for the biosynthesis of aurofusarin in Fusarium graminearum. J. Biol. Chem. 2011, jbc. M110. 179853.

(5). Gil Girol, C.; Fisch, K. M.; Heinekamp, T.; Günther, S.; Hüttel, W.; Piel, J.; Brakhage, A. A.; Müller, M., Regio - and stereoselective oxidative phenol coupling in Aspergillus niger. Angew. Chem., Int. Ed. 2012, 51 (39), 9788-9791.
(6). Mazzaferro, L. S.; Hüttel, W.; Fries, A.; Müller, M., Cytochrome P450-catalyzed regio-and stereoselective phenol coupling of fungal natural products. J. Am. Chem. Soc. 2015, 137 (38), 12289-12295.

(7). Fang, W.; Ji, S.; Jiang, N.; Wang, W.; Zhao, G. Y.; Zhang, S.; Ge, H. M.; Xu, Q.; Zhang, A. H.; Zhang, Y. L.; Song, Y. C.; Zhang, J.; Tan, R. X., Naphthol radical couplings determine structural features and enantiomeric excess of dalesconols in Daldinia eschscholzii. Nat Commun. 2012, 3, 1039.

(8). Davin, L. B.; Wang, H.-B.; Crowell, A. L.; Bedgar, D. L.; Martin, D. M.; Sarkanen, S.; Lewis, N. G., Stereoselective bimolecular phenoxy radical coupling by an auxiliary (dirigent) protein without an active center. Science 1997, 275 (5298), 362-367.

(9). Liu, J.; Stipanovic, R. D.; Bell, A. A.; Puckhaber, L. S.; Magill, C. W., Stereoselective coupling of hemigossypol to form (+)-gossypol in moco cotton is mediated by a dirigent protein. Phytochemistry 2008, 69 (18), 3038-3042.

(10). Pickel, B.; Schaller, A., Dirigent proteins: molecular characteristics and potential biotechnological applications. Appl. Microbiol. Biotechnol. 2013, 97 (19), 8427-8438.

(11). Hu, J.; Sarrami, F.; Li, H.; Zhang, G.; Stubbs, K. A.; Lacey, E.; Stewart, S. G.; Karton, A.; Piggott, A. M.; Chooi, Y.-H., Heterologous biosynthesis of elsinochrome A sheds light on the formation of the photosensitive perylenequinone system. Chem. Sci. 2019, 10, $1457-$ 1465.

(12). Lillehoj, E.; Milburn, M., Viriditoxin production by Aspergillus viridi-nutans and related species. Appl. Microbiol. 1973, 26 (2), 202-205

(13). Suzuki, K.; Nozawa, K.; Nakajima, S.; Kawai, K., Structure revision of mycotoxin, viriditoxin, and its derivatives. Chem. Pharm Bull. 1990, 38 (11), 3180-3181.

(14). Mizuba, S.; Hsu, C.; Jiu, J., A third metabolite from Spicaria divaricata NRRL 5771. J. Antibiot. 1977, 30 (8), 670-672.

(15). Jiu, J.; MIZUBA, S., Metabolic products from Spicaria divaricata NRRL 5771. J. Antibiot. 1974, 27 (10), 760-765.

(16). Ayer, W. A.; Craw, P. A.; Nozawa, K., Two 1 H-naphtho [2, 3c] pyran-1-one metabolites from the fungus Paecilomyces variotii. Can. J. Chem. 1991, 69 (2), 189-191.

(17). Wang, J.; Galgoci, A.; Kodali, S.; Herath, K. B.; Jayasuriya, H.; Dorso, K.; Vicente, F.; González, A.; Cully, D.; Bramhill, D., Discovery of a small molecule that inhibits cell division by blocking FtsZ, a novel therapeutic target of antibiotics. J. Biol. Chem. 2003, 278 (45), 4442444428.

(18). Liu, Y.; Kurtán, T.; Wang, C. Y.; Lin, W. H.; Orfali, R.; Müller, W. E.; Daletos, G.; Proksch, P., Cladosporinone, a new viriditoxin derivative from the hypersaline lake derived fungus Cladosporium cladosporioides. J. Antibiot. 2016, 69 (9), 702.

(19). Kundu, S.; Kim, T. H.; Yoon, J. H.; Shin, H.-S.; Lee, J.; Jung, J. H.; Kim, H. S., Viriditoxin regulates apoptosis and autophagy via mitotic catastrophe and microtubule formation in human prostate cancer cells. Int. J. Oncol. 2014, 45 (6), 2331-2340.

(20). Anderson, D. E.; Kim, M. B.; Moore, J. T.; O’Brien, T. E.; Sorto, N. A.; Grove, C. I.; Lackner, L. L.; Ames, J. B.; Shaw, J. T., Comparison of small molecule inhibitors of the bacterial cell division protein FtsZ and identification of a reliable cross-species inhibitor. ACS Chem. Biol. 2012, 7 (11), 1918-1928.

(21). Park, Y. S.; Grove, C. I.; González - López, M.; Urgaonkar, S.; Fettinger, J. C.; Shaw, J. T., Synthesis of (-) - Viriditoxin: A 6, 6' Binaphthopyran - 2 - one that Targets the Bacterial Cell Division Protein FtsZ. Angew. Chem., Int. Ed. 2011, 50 (16), 3730-3733.

(22). Tan, N. P.; Donner, C. D., Total synthesis and confirmation of the absolute stereochemistry of semiviriditoxin, a naphthopyranone metabolite from the fungus Paecilomyces variotii. Tetrahedron 2009 65 (20), 4007-4012.

(23). Urquhart, A.; Mondo, S.; Mäkelä, M.; Hane, J.; Wiebenga, A.; He, G.; Mihaltcheva, S.; Pangilinan, J.; Lipzen, A.; Barry, K., Genomic and genetic insights into a cosmopolitan fungus, Paecilomyces variotii (Eurotiales). Front. Microbiol. 2018, 9, 3058.

(24). Chooi, Y. H.; Zhang, G.; Hu, J.; Muria - Gonzalez, M. J.; Tran, P. N.; Pettitt, A.; Maier, A. G.; Barrow, R. A.; Solomon, P. S., Functional genomics - guided discovery of a light - activated phytotoxin in the wheat pathogen Parastagonospora nodorum via pathway activation. Environ. Microbiol. 2017, 19 (5), 1975-1986. 
(25). Fiorentini, F.; Romero, E.; Fraaije, M. W.; Faber, K.; Hall, M.; Mattevi, A., Baeyer-Villiger Monooxygenase FMO5 as Entry Point in Drug Metabolism. ACS Chem. Biol. 2017, 12 (9), 2379-2387.

(26). van Beek, H. L.; Romero, E.; Fraaije, M. W., Engineering cyclohexanone monooxygenase for the production of methyl propanoate. ACS Chem. Biol. 2016, 12 (1), 291-299.

(27). Chiang, Y. M.; Ahuja, M.; Oakley, C. E.; Entwistle, R.; Asokan, A.; Zutz, C.; Wang, C. C.; Oakley, B. R., Development of genetic dereplication strains in Aspergillus nidulans results in the discovery of aspercryptin. Angew. Chem., Int. Ed. 2016, 55 (5), 1662-1665.

(28). Tolmie, C.; Smit, M. S.; Opperman, D. J., Native roles of Baeyer-Villiger monooxygenases in the microbial metabolism of natural compounds. Nat. Prod. Rep. 2019, 36, 326-353.

(29). Wang, G.-Q.; Chen, G.-D.; Qin, S.-Y.; Hu, D.; Awakawa, T.; Li S.-Y.; Lv, J.-M.; Wang, C.-X.; Yao, X.-S.; Abe, I.; Gao, H., Biosynthetic pathway for furanosteroid demethoxyviridin and identification of an unusual pregnane side-chain cleavage. Nat. Commun. 2018, 9 (1), 1838.

(30). Cygler, M.; Grochulski, P.; Kazlauskas, R. J.; Schrag, J. D.; Bouthillier, F.; Rubin, B.; Serreqi, A. N.; Gupta, A. K., A structural basis for the chiral preferences of lipases. J. Am. Chem. Soc. 1994, 116 (8), 3180-3186

(31). Li, G.; Garcia-Borràs, M.; Fürst, M. J.; Ilie, A.; Fraaije, M. W. Houk, K. N.; Reetz, M. T., Overriding Traditional Electronic Effects in Biocatalytic Baeyer-Villiger Reactions by Directed Evolution. J. Am. Chem. Soc. 2018, 140 (33), 10464-10472.

(32). Hu, Y.; Dietrich, D.; Xu, W.; Patel, A.; Thuss, J. A.; Wang, J.; Yin, W.-B.; Qiao, K.; Houk, K. N.; Vederas, J. C., A carbonate-forming Baeyer-Villiger monooxygenase. Nat. Chem. Biol. 2014, 10 (7), 552.

(33). Fürtges, L.; Obermaier, S.; Thiele, W.; Foegen, S.; Müller, M., Diversity in Fungal Intermolecular Phenol Coupling of Polyketides Regioselective Laccase - based systems. ChemBioChem 2019. DOI: $10.1002 /$ cbic. 201900041

(34). Gasper, R.; Effenberger, I.; Kolesinski, P.; Terlecka, B. Hofmann, E.; Schaller, A., Dirigent protein mode of action revealed by the crystal structure of AtDIR6. Plant Physiol. 2016, pp. 01281.2016.

(35). Kim, K.-W.; Smith, C. A.; Daily, M. D.; Cort, J. R.; Davin, L. B.; Lewis, N. G., Trimeric structure of (+)-pinoresinol-forming dirigent protein at $1.95 \AA$ resolution with three isolated active sites. J. Biol. Chem. 2015, 290 (3), 1308-1318.

(36). Sato, M.; Yagishita, F.; Mino, T.; Uchiyama, N.; Patel, A.; Chooi, Y. H.; Goda, Y.; Xu, W.; Noguchi, H.; Yamamoto, T., Involvement of Lipocalin - like CghA in Decalin - Forming Stereoselective Intramolecular [4+ 2] Cycloaddition. ChemBioChem 2015, 16 (16), 2294-2298.

(37). Kato, N.; Nogawa, T.; Takita, R.; Kinugasa, K.; Kanai, M.; Uchiyama, M.; Osada, H.; Takahashi, S., Control of the Stereochemical Course of [4+2] Cycloaddition during trans - Decalin Formation by Fsa2 - Family Enzymes. Angew. Chem. 2018, 130 (31), 9902-9906.

(38). Jamieson, C. S.; Ohashi, M.; Liu, F.; Tang, Y.; Houk, K., The expanding world of biosynthetic pericyclases: cooperation of experiment and theory for discovery. Nat. Prod. Rep. 2019. DOI: 10.1039/C8NP00075A

(39). Tan, D.; Jamieson, C. S.; Ohashi, M.; Tang, M.-C.; Houk, K. N.; Tang, Y., Genome-mined Diels-Alderase catalyzes formation of the cisoctahydrodecalins of varicidin A and B. J. Am. Chem. Soc. 2019, 141 (2), 769-773.

(40). Li, L.; Yu, P.; Tang, M.-C.; Zou, Y.; Gao, S.-S.; Hung, Y.-S.; Zhao, M.; Watanabe, K.; Houk, K.; Tang, Y., Biochemical characterization of a eukaryotic decalin-forming Diels-Alderase. J. Am. Chem. Soc. 2016, 138 (49), 15837-15840. 
VdtE: Baeyer-Villiger monooxygenase

VdtD: hydrolase-like dirigent protein

(1:2 ratio) 